Service social

\title{
L'évaluation des services, des programmes et des politiques. Pour améliorer la qualité des services
}

\section{Marcel Sénéchal et Mireille Tremblay}

Volume 47, numéro 1-2, 1998

Évaluation - Colloque 1999

URI : https://id.erudit.org/iderudit/706777ar

DOI : https://doi.org/10.7202/706777ar

Aller au sommaire du numéro

Éditeur(s)

École de service social de l'Université Laval

ISSN

1708-1734 (numérique)

Découvrir la revue

Citer ce document

Sénéchal, M. \& Tremblay, M. (1998). L'évaluation des services, des programmes et des politiques. Pour améliorer la qualité des services. Service social, 47(1-2),

9-10. https://doi.org/10.7202/706777ar d'utilisation que vous pouvez consulter en ligne. 


\section{L'évaluation des services, des programmes et des politiques Pour améliorer la qualité des services}

L'évaluation des services au niveau local, des programmes au niveau régional et des politiques au niveau provincial a connu un essor important dans le réseau de la santé et des services sociaux au Québec. Alors que dans la plupart des documents qui ont émaillé la réforme des services de santé et des services sociaux au début des années 1990 on déplorait la méconnaissance des services et l'absence d'évaluation quant à leur qualité et à leur impact sur l'état de santé de la population, des efforts ont été déployés tant au sein du ministère de la Santé et des Services sociaux que par les régies régionales et les établissements pour améliorer nos outils d'évaluation. Plusieurs des outils qui nous faisaient défaut il y a dix ans sont aujourd'hui au rendez-vous : programmes et politiques mieux définis, modèles d'analyse et expertise en évaluation de programmes, systèmes d'information, structures de recherche, mandats dévolus aux instances provinciales, régionales et locales. Or, malgré toutes les pratiques qui ont été mises en œuvre, l'évaluation n'a pas encore répondu aux attentes des divers groupes d'acteurs.

C'est pour faire le point sur cette question que le Conseil québécois d'agrément et la Fédération québécoise des CRPDI conviaient leurs partenaires du réseau de la santé et des services sociaux à une journée de réflexion sur l'évaluation des services, des programmes et des politiques, en juin 1999. Si nous disposons à l'heure actuelle des outils méthodologiques et techniques pour évaluer les services, les programmes et les politiques, ces outils sont peu diffusés et les résultats sont insuffisamment utilisés par les divers groupes d'acteurs, décideurs, 
planificateurs, intervenants et usagers. Les participants et participantes au colloque ont insisté sur la nécessité de diffuser les outils et les résultats disponibles ainsi que sur le besoin d'améliorer la coordination de l'évaluation entre les niveaux local, régional et provincial. Ils ont été unanimes quant à l'utilité de l'évaluation qui est indispensable à l'amélioration de la qualité des services, des programmes et des politiques. Certains ont même suggéré que des budgets protégés lui soient réservés.

Afin de partager les résultats de cette réflexion, un numéro spécial de la revue Service social est consacré à ce thème. Vous y trouverez donc un résumé des débats, un certain nombre de conférences présentées durant cette journée, de même qu'une analyse des enjeux politiques et méthodologiques rédigée par deux membres du comité organisateur. Pour enrichir la réflexion sur la question de l'évaluation et en cerner les principaux éléments, une série d'articles a été ajoutée aux actes du colloque. Tous les textes qui suivent vous proposent des outils de travail et de réflexion sur la pratique évaluative dans le réseau de la santé et des services sociaux au Québec. Nous profitons de l'occasion pour remercier tous les membres du comité organisateur, les participants et participantes au colloque et les auteurs qui ont bien voulu contribuer à cette publication.

Marcel SÉNÉCHAL Mireille TREMBLAY 